\title{
Tratamento da síndrome do túnel ulnar pela técnica da epicondilectomia parcial medial do cotovelo*
}

\author{
Treatment of cubital tunnel syndrome using the technique \\ of medial partial epicondylectomy of the elbow
}

\author{
Marcio Eduardo de Melo Viveiros ${ }^{1}$, Marcelo Hide Matsumoto², Thiago Bernardes Bastos ${ }^{3}$, \\ Marcio José Soares de Moraes ${ }^{4}$, Anderson Uehara ${ }^{5}$, Henrique Pereira ${ }^{6}$
}

\section{RESUMO}

Objetivo: Analisamos retrospectivamente os resultados de 21 casos de síndrome cubital tratados cirurgicamente com a técnica da epicondilectomia parcial medial. Métodos: No período de fevereiro de 2001 a outubro de 2006, 21 pacientes com síndrome do canal cubital foram tratados pela técnica da epicondilectomia parcial medial do cotovelo associada à neurólise do nervo ulnar. Destes, 12 $(57,1 \%)$ eram do sexo masculino. $O$ lado direito foi o acometido em $15(71,4 \%)$ pacientes. A média da idade dos pacientes foi de 51,6 anos. Pela graduação de McGowan, seis $(\mathbf{2 8 , 6 \% )}$ pacientes encontravam-se no grau I, $11(52,3 \%)$, no grau II e quatro
(19,1\%), no grau III do período pré-operatório. Resultados: O tempo médio de acompanhamento pós-operatório foi de 25,7 meses. No pós-operatório, os pacientes foram avaliados conforme a escala de pontos de Bishop, sendo que nove $(42,8 \%)$ apresentavam resultados excelentes, sete $(33,3 \%)$, bons, três $(14,2 \%)$, regulares e dois $(9,5 \%)$, ruins. Nesta série, não se encontraram como complicações a instabilidade em valgo residual, a lesão permanente do nervo ulnar, a recidiva da compressão ou a subluxação do nervo ulnar. As complicações encontradas foram perda do arco de movimento em um $(4,7 \%)$ caso, infecção superficial em um $(4,7 \%)$ e um $(4,7 \%)$ com dor residual. Conclusão: Os resultados apresentados permitem concluir que a epi-
* Trabalho realizado no Grupo de Cotovelo da Disciplina de Cirurgia da Mão e Membro Superior do Departamento de Ortopedia e Traumatologia da Escola Paulista de Medicina da Universidade Federal de São Paulo - UNIFESP - São Paulo (SP), Brasil.

1. Mestre, Médico Assistente no Grupo de Ombro e Cotovelo da Disciplina de Cirurgia da Mão e Membro Superior do Departamento de Ortopedia e Traumatologia da Escola Paulista de Medicina da Universidade Federal de São Paulo - UNIFESP - São Paulo (SP), Brasil.

2. Doutor, Chefe do Grupo de Ombro e Cotovelo da Disciplina de Cirurgia da Mão e Membro Superior do Departamento de Ortopedia e Traumatologia da Escola Paulista de Medicina da Universidade Federal de São Paulo - UNIFESP - São Paulo (SP), Brasil.

3. Residente do Terceiro Ano do Departamento de Ortopedia e Traumatologia do Departamento de Ortopedia e Traumatologia da Escola Paulista de Medicina da Universidade Federal de São Paulo - UNIFESP - São Paulo (SP), Brasil.
4. Especializando do Grupo de Ombro e Cotovelo do Departamento de Ortopedia e Traumatologia do Departamento de Ortopedia e Traumatologia da Escola Paulista de Medicina da Universidade Federal de São Paulo - UNIFESP - São Paulo (SP), Brasil.

5. Médico Assistente no Grupo de Cotovelo da Disciplina de Cirurgia da Mão e Membro Superior do Departamento de Ortopedia e Traumatologia da Escola Paulista de Medicina da Universidade Federal de São Paulo - UNIFESP - São Paulo (SP), Brasil.

6. Médico Assistente no Grupo de Cotovelo da Disciplina de Cirurgia da Mão e Membro Superior do Departamento de Ortopedia e Traumatologia da Escola Paulista de Medicina da Universidade Federal de São Paulo - UNIFESP - São Paulo (SP), Brasil.

Endereço para correspondência: Rua Borges Lagoa, 783, 5o andar - 04038-001 - São Paulo (SP) - Brasil. Tel.: (11) 5571-7106.

E-mail: bernardesthiago@terra.com.br

Recebido: 10/12/07. Revisão: 6/3/08. Reapresentação: 23/9/08. Aprovado: 27/11/08.

Copyright RBO2008 
condilectomia parcial medial do cotovelo associada à neurólise do nervo ulnar é eficiente e segura para o tratamento da síndrome do canal cubital.

\author{
Descritores - Síndrome do túnel ulnar/cirurgia; Cotovelo de \\ tenista/cirurgia; Estudos retrospectivos
}

\section{ABSTRACT}

Objective: The authors made a retrospective analysis of the results of 21 cases of cubital syndrome that were surgically treated with the partial medial epicondylectomy. Methods: From February 2001 to October 2006, 21 patients with cubital tunnel syndrome were treated with the technique of elbow partial medial epicondylectomy associated to neurolysis of the ulnar nerve. Of these patients, $12(57.1 \%)$ were male. The right side was involved in 15 (71.4\%). Mean age of the patients was 51.6 years. According to McGowan classification, six (28.6\%) patients were grade I, 11 (52.3\%), grade II, and four (19.1\%), grade III in the preoperative period. Results: The mean post-operative follow-up time was 25.7 months. In the post-operative period, patients were evaluated according to the Bishop score scale, and nine of them (42.8\%) showed excellent results, seven (33.3\%) had good results, three (14.2\%), regular, and two patients (9.5\%) had poor results. The authors did not find in this series any complications represented by residual valgus instability, permanent lesion of the ulnar nerve, recurrent compression, or subluxation of the ulnar nerve. Complications found were the loss of the movement arch in one case (4.7\%), superficial infection in one case (4.7\%), and residual pain in one case (4.7\%). Conclusion: The results allow for the conclusion that partial medial epicondylectomy of the elbow associated to neurolysis of the ulnar nerve is effective and safe to treat cubital tunnel syndrome.

Keywords - Cubital tunnel syndrome/surgery; Tennis elbow/surgery; Retrospective studies

\section{INTRODUÇÃO}

O nervo ulnar, em seu trajeto pelo membro superior, pode sofrer compressão em vários níveis, sendo o cotovelo o local mais frequiente. A síndrome do túnel cubital, como é denominada essa alteração, é a segunda neuropatia compressiva mais comum do membro superior, após a síndrome do túnel do carpo ${ }^{(1)}$. O trajeto anatômico do nervo ulnar no cotovelo é complexo, existindo uma relação entre as variações anatômicas do ligamento retinacular cubital com a compressão ner$\operatorname{vosa}^{(2)}$.

Apferg et al relataram diversos fatores que podem ocasionar a neuropatia ulnar no cotovelo, entre elas, as sequielas das fraturas-luxações no cotovelo, as anomalias congênitas e as alterações metabólicas ${ }^{(3)}$. Porém, a causa primária da compressão é indeterminada em $10 \%$ a $30 \%$ dos casos. As alterações sistêmicas comumente associadas aos indivíduos sintomáticos são a diabetes mellitus (DM), as doenças renais, o mieloma múltiplo, a amiloidose, o etilismo crônico, a desnutrição e a hanseníase ${ }^{(3)}$.

O quadro clínico é caracterizado por dor, parestesia e hipoestesia de caráter intermitente no território do nervo ulnar, que se acentuam com a abdução do ombro, a flexão do cotovelo e no período noturno. Quando o cotovelo é fletido, a origem do músculo flexor ulnar do carpo é tensionada, alongando aproximadamente $5 \mathrm{~mm}$ a cada $45^{\circ}$ de flexão, e o ligamento colateral medial se projeta medialmente, diminuindo o túnel cubital e, assim, produzindo sintomas de neuropatia do ulnar ${ }^{(1)}$. Diversos sinais e sintomas nos auxiliam na diferenciação da síndrome do túnel cubital da síndrome do canal de Guyon. Dentre eles podemos citar: dor na face medial do cotovelo, piora dos sintomas com a flexão do cotovelo, parestesia na face dorsal da mão, redução da força dos flexores profundos do anular e dedo mínimo e do flexor ulnar do carpo ${ }^{(4)}$. Ocasionalmente, verifica-se o comprometimento motor, que é evidenciado pela hipotrofia da musculatura intrínseca da mão e, nos casos mais avançados, comprometimento da musculatura extrínseca.

Os diagnósticos diferenciais da síndrome cubital são a síndrome do canal de Guyon, lesão do disco cervical, costela cervical, síndrome do escaleno anterior, tumor de Pancoast, doenças metabólicas, doença de Hansen e neuropatia alcoólica ${ }^{(1)}$.

O tratamento cirúrgico da síndrome do túnel cubital inclui a descompressão isolada, a descompressão associada à transposição anterior do nervo ulnar (sub- 
muscular, intramuscular ou subcutânea) ou a epicondilectomia medial ${ }^{(5-6)}$.

O objetivo do presente estudo é a análise retrospectiva da neurólise do nervo ulnar associada à epicondilectomia parcial medial como tratamento da síndrome cubital.

\section{MÉTODOS}

No período de fevereiro de 2001 a outubro de 2006 foram tratados 21 pacientes (21 cotovelos) com síndrome do túnel ulnar pela técnica de neurólise do nervo ulnar associada à epicondilectomia parcial medial do cotovelo ${ }^{(6)}$, no Instituto da Mão UNIFESP, São Paulo, SP. Foram incluídos pacientes refratários por seis meses ao tratamento não cirúrgico e eletroneuromiografia compatível com síndrome do túnel cubital. Fatores de exclusão foram: procedimento cirúrgico prévio para tratamento da síndrome do túnel cubital e sequiela de fratura do cotovelo.
Dos pacientes, 12 (57\%) eram do sexo masculino e nove $(43 \%)$, do feminino; O lado direito foi acometido em $15(71,4 \%)$ pacientes e o esquerdo em seis (28,6\%). A idade variou de 18 anos a 67 anos, com média de 51,6 anos. O tempo de início dos sintomas foi, em média, de 11 meses, variando de quatro a 24 meses. Encontramos associação com trauma no cotovelo em $33 \%$ dos casos.

O tempo de seguimento pós-operatório variou de 52 meses até 12 meses (tabela 1).

\section{Técnica cirúrgica}

Os procedimentos foram realizados sob anestesia locorregional (bloqueio de plexo braquial). Os pacientes posicionados em supino com o membro superior acometido em abdução e rotação lateral sobre a tábua de mão. Realizada incisão curvilínea na pele ao longo do trajeto do nervo ulnar centrada sobre a face poste-

TABELA 1

Distribuição dos pacientes em numeração, sexo, idade, lado acometido, tempo de sintomas em meses, classificação segundo McGowan, afeç̧ões associadas e tempo de seguimento em meses

\begin{tabular}{|c|c|c|c|c|c|c|c|}
\hline ID & Sexo & Idade / anos & Lado & $\begin{array}{c}\text { Tempo dos } \\
\text { sintomas/meses }\end{array}$ & McGowan & $\begin{array}{c}\text { Afecções } \\
\text { associadas }\end{array}$ & $\begin{array}{c}\text { Tempo de } \\
\text { seguimento } \\
\text { em meses }\end{array}$ \\
\hline 1 & M & 18 & $\mathrm{D}$ & 6 & II & Traumática & 58 \\
\hline 2 & $M$ & 39 & $\mathrm{D}$ & 11 & II & Hansen & 36 \\
\hline 3 & M & 39 & $\mathrm{E}$ & 18 & III & Idiopática & 15 \\
\hline 4 & $F$ & 43 & $E$ & 9 & 1 & Idiopática & 19 \\
\hline 5 & $\mathrm{~F}$ & 55 & $\mathrm{D}$ & 13 & I & Idiopática & 40 \\
\hline 6 & $\mathrm{~F}$ & 56 & $\mathrm{D}$ & 14 & II & Idiopática & 20 \\
\hline 7 & M & 60 & $\mathrm{D}$ & 8 & I & Luxação do cotovelo & 30 \\
\hline 8 & $\mathrm{~F}$ & 59 & $\mathrm{D}$ & 8 & I & Idiopática & 24 \\
\hline 9 & M & 55 & $\mathrm{D}$ & 24 & II & Traumática & 24 \\
\hline 10 & M & 64 & $\mathrm{E}$ & 13 & II & Idiopática & 37 \\
\hline 11 & M & 63 & $\mathrm{D}$ & 8 & III & Pseudartrose & 32 \\
\hline 12 & $\mathrm{~F}$ & 55 & $E$ & 7 & 1 & Idiopática & 12 \\
\hline 13 & $M$ & 45 & $\mathrm{D}$ & 20 & II & Traumática & 20 \\
\hline 14 & M & 51 & $\mathrm{D}$ & 6 & II & Idiopática & 23 \\
\hline 15 & $M$ & 67 & $\mathrm{D}$ & 9 & II & Idiopática & 25 \\
\hline 16 & M & 53 & $\mathrm{E}$ & 13 & 1 & Idiopática & 20 \\
\hline 17 & $F$ & 62 & $E$ & 21 & III & Idiopática & 21 \\
\hline 18 & $\mathrm{~F}$ & 53 & $\mathrm{D}$ & 8 & II & Idiopática & 19 \\
\hline 19 & $\mathrm{~F}$ & 49 & $\mathrm{D}$ & 9 & II & Traumática & 25 \\
\hline 20 & $\mathrm{~F}$ & 50 & $\mathrm{D}$ & 12 & II & Idiopática & 21 \\
\hline 21 & M & 49 & $\mathrm{D}$ & 4 & III & Trauma & 20 \\
\hline
\end{tabular}

$M=$ masculino; $F=$ feminino; $D=$ direito; $E$ = esquerdo

Fonte: Universidade Federal de São Paulo - DOT - EPM 2008 
rior do epicôndilo medial (figuras 1 e 2). Realizada dissecção cautelosa até a fáscia profunda, com o objetivo de não lesarmos os ramos do nervo cutâneo medial do braço e do nervo cutâneo medial de antebraço. Realizada exposição subperiosteal do epicôndilo medial após incisão longitudinal na origem da musculatura flexopronadora, preservando o ligamento colateral medial. Identificado o nervo ulnar, com auxílio de uma "fita cardíaca"; realizada, em seguida, a liberação deste nos cinco prováveis pontos de compressão, de proximal para distal (figuras 3 e 4). A seguir, foi realizada a osteotomia do epicôndilo medial, de distal

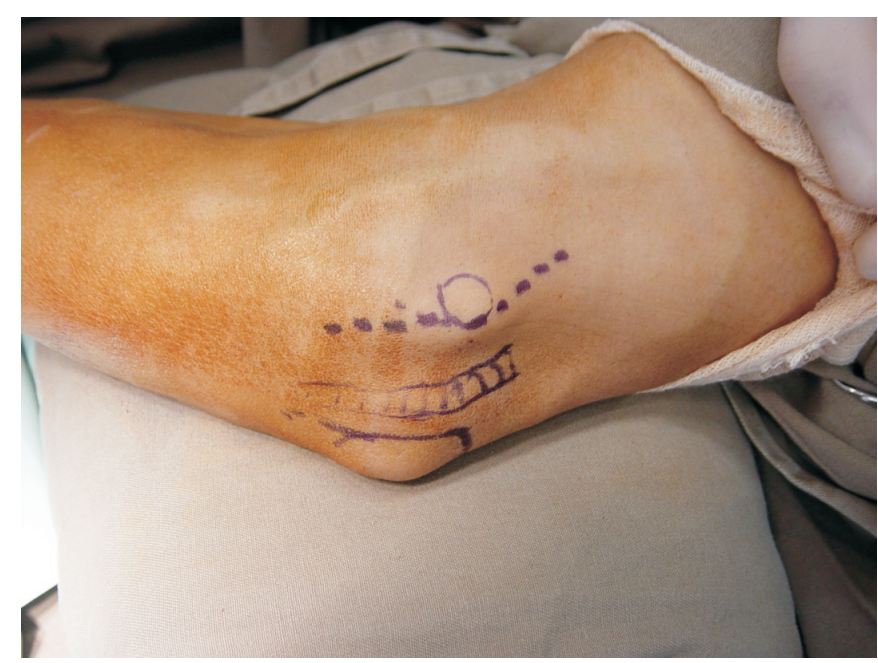

Figura 1 - Relação da incisão curvilínea com o epicôndilo medial e com o nervo ulnar

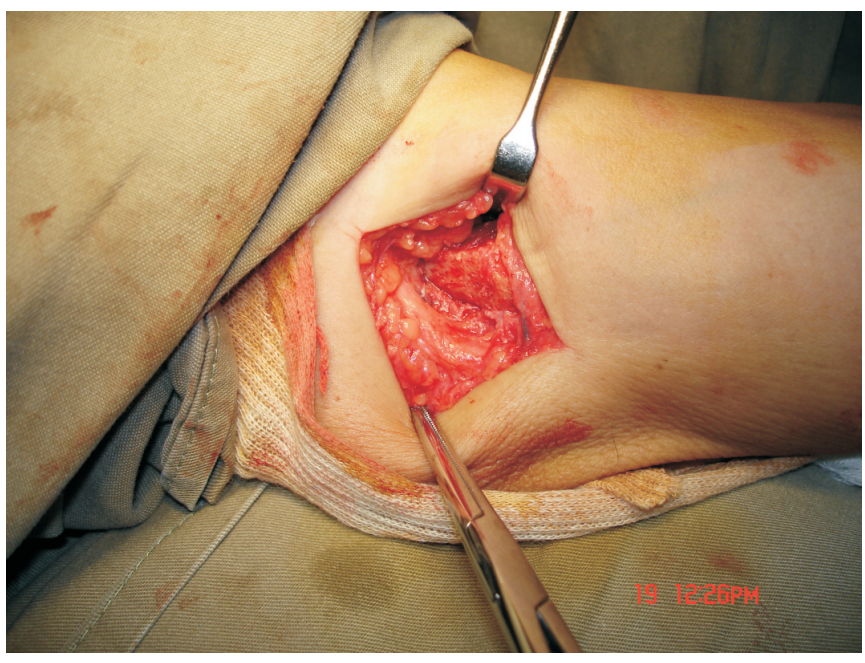

Figura 3 - Identificação do nervo ulnar para proximal (figura 5). A quantidade de osso retirada deve ser suficiente para permitir a translação anterior do nervo ulnar quando da flexão; todavia, não pode ser excessiva, a fim de preservamos a origem do ligamento colateral medial (figura 6). Em média, são retirados de 7 a $8 \mathrm{~mm}$ de largura do epicôndilo medial (figura 7). Em seguida, efetua-se a regularização das superfícies ósseas da osteotomia. Sutura do periósteo com Poliglactina 910 (vicryl $^{\circledR}$ ) 3.0 (figura 8). Mobilização da articulação em todo o arco de movimento. Liberação do torniquete e hemostasia. Não utilizamos imobilização no pós-operatório.

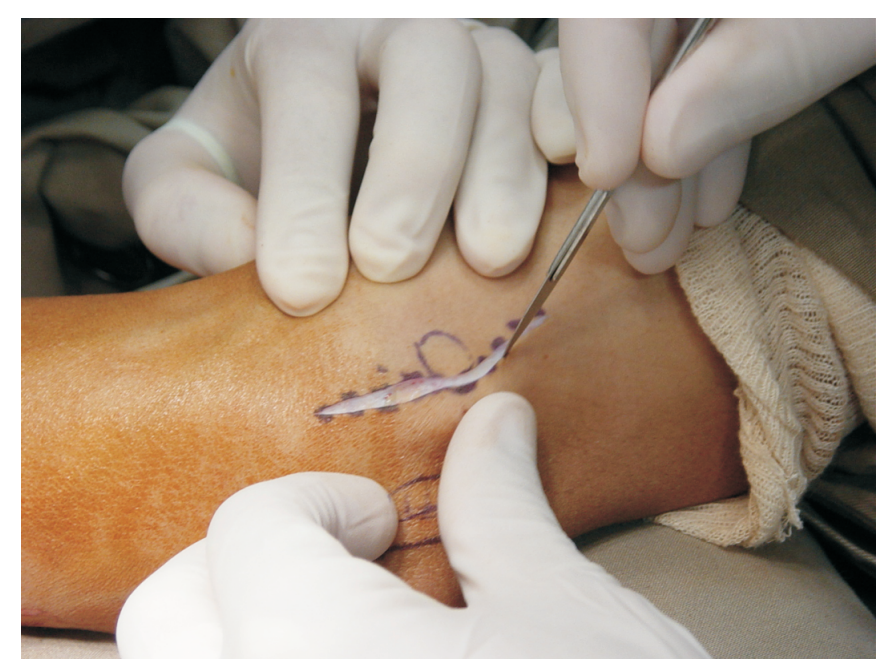

Figura 2 - Realização da via sobre a linha previamente desenhada

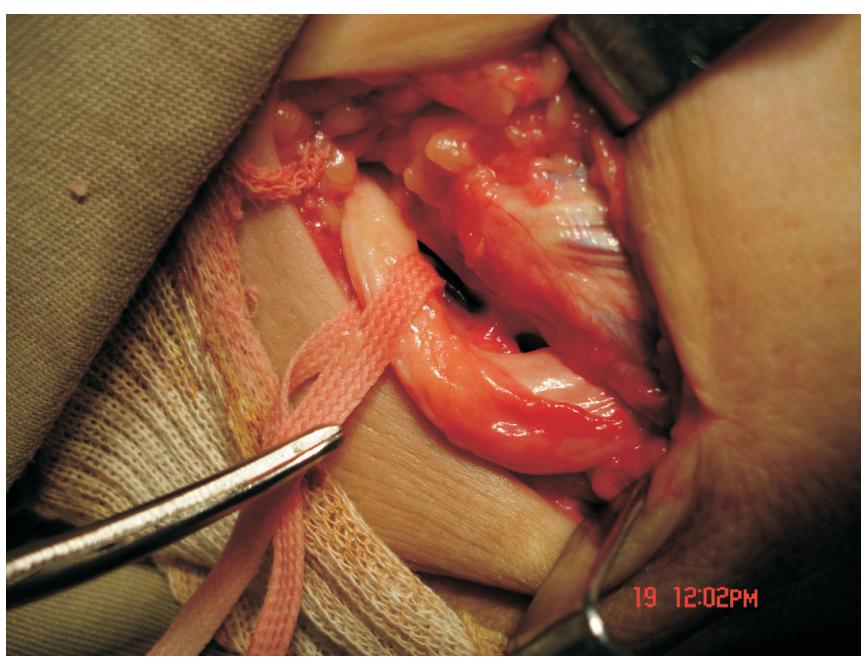

Figura 4 - Isolado nervo ulnar com auxílio de uma fita cardíaca 


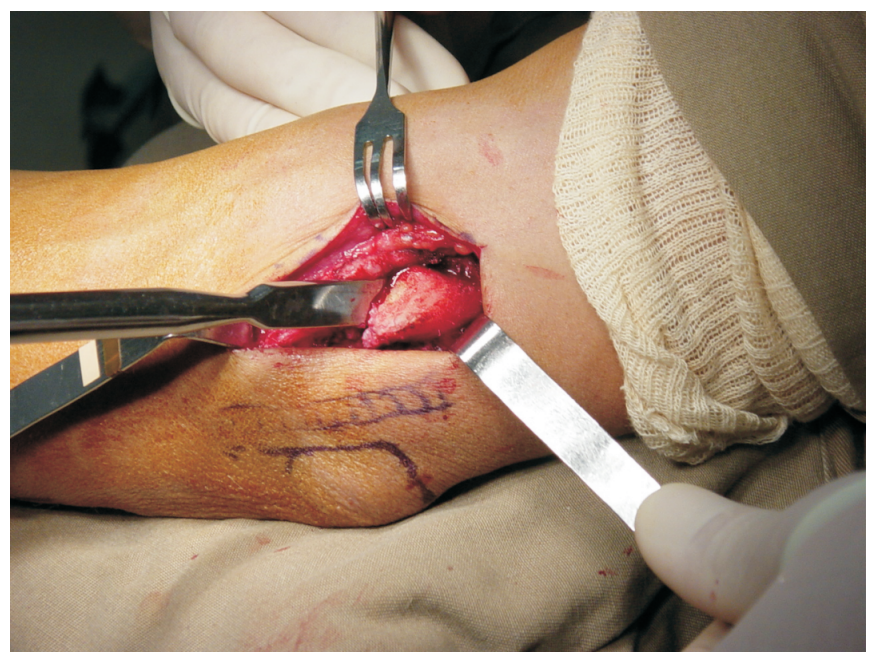

Figura 5 - Realização da osteotomia parcial do epicôndilo medial de distal para proximal

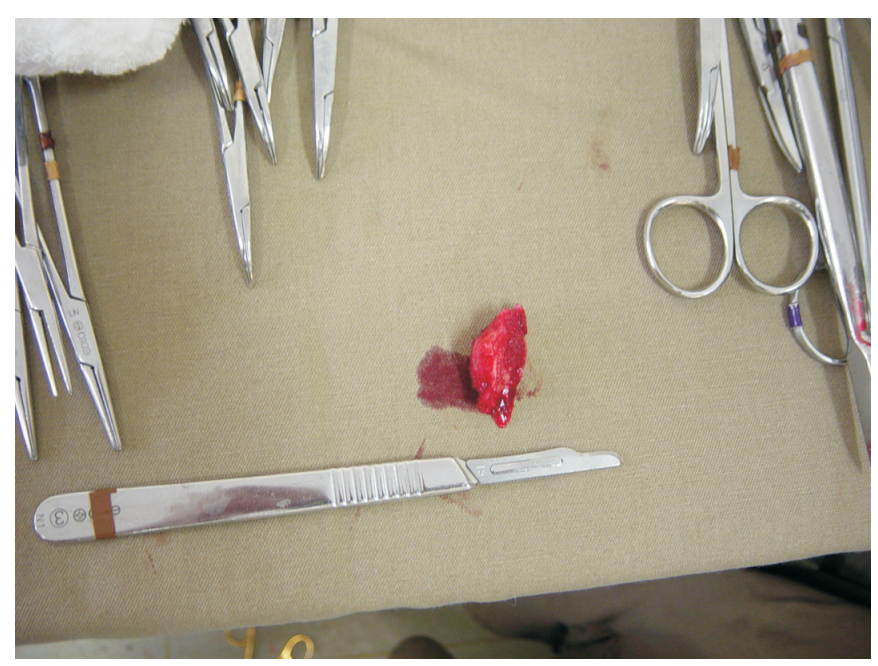

Figura 7 - Fragmento do epicôndilo medial retirado: em média, 7 a $8 \mathrm{~mm}$

\section{RESULTADOS}

Encontramos pela classificação de $\mathrm{McGowan}^{(7)}$ seis pacientes grau I, 11 pacientes grau II e quatro pacientes grau III (gráfico 1).

Os resultados obtidos foram pontuados conforme a escala conhecida como de Bishop $^{(8)}$ (tabela 2 e gráfico 2).

\section{Complicações}

Identificamos um paciente $(4,7 \%)$ com infecção superficial, um $(4,7 \%)$ com dor residual e um $(4,7 \%)$ com diminuição do arco de movimento.

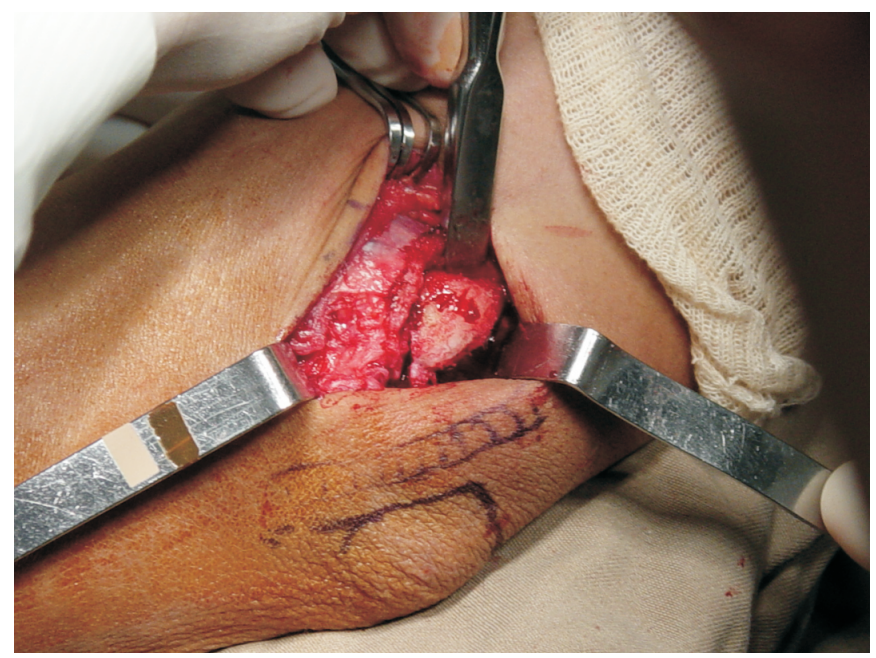

Figura 6 - Epicôndilo medial após a retirada do fragmento e regularização das bordas da osteotomia

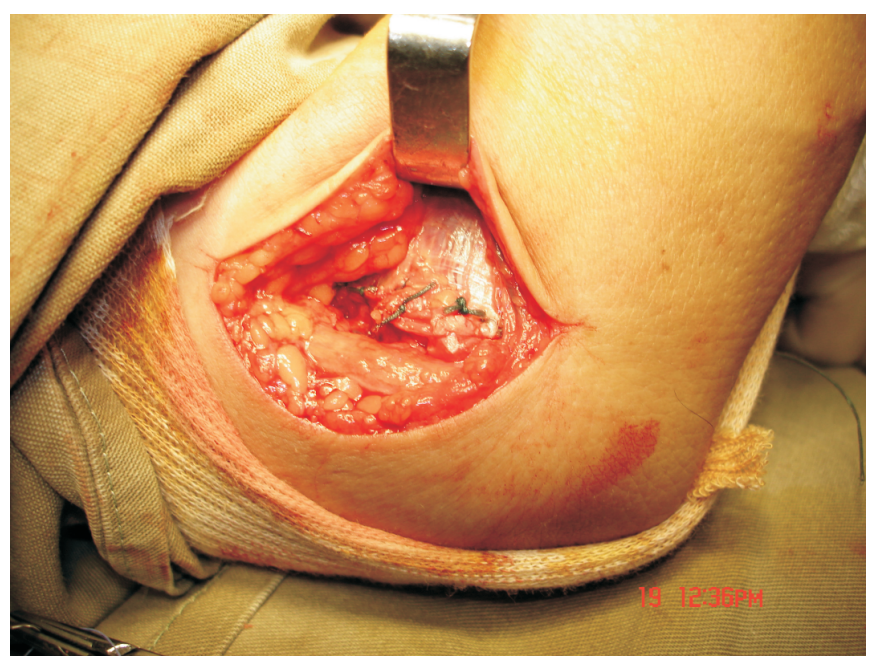

Figura 8 - Sutura do periósteo com vicryl 3.0

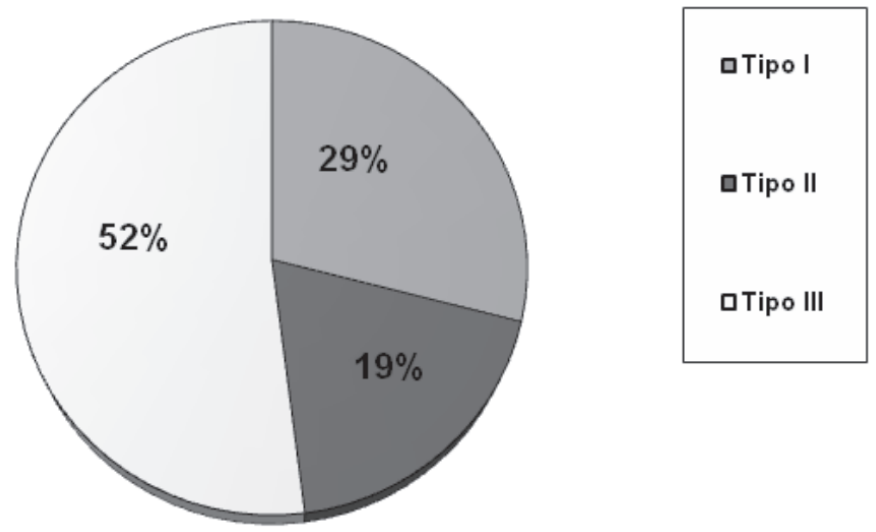

Gráfico 1 - Distribuição dos pacientes segundo a classificação de McGowan

Fonte: Universidade Federal de São Paulo - DOT - EPM 2008 
TABELA 2

Pontuação dos pacientes segundo a avaliação do sistema de Bishop

\begin{tabular}{crl}
\hline Número & Pontuação & Resultados \\
\hline 1 & 11 & Excelente \\
2 & 2 & Ruim \\
3 & 10 & Excelente \\
4 & 6 & Regular \\
5 & 9 & Bom \\
6 & 10 & Excelente \\
7 & 9 & Bom \\
8 & 9 & Bom \\
9 & 11 & Excelente \\
10 & 9 & Bom \\
11 & 11 & Excelente \\
12 & 5 & Regular \\
13 & 10 & Excelente \\
14 & 10 & Excelente \\
15 & 9 & Bom \\
16 & 11 & Excelente \\
17 & 6 & Regular \\
18 & 9 & Bom \\
19 & 11 & Excelente \\
20 & 9 & Bom \\
21 & 3 & Ruim \\
\hline
\end{tabular}

Fonte: Universidade Federal de São Paulo - DOT - EPM 2008

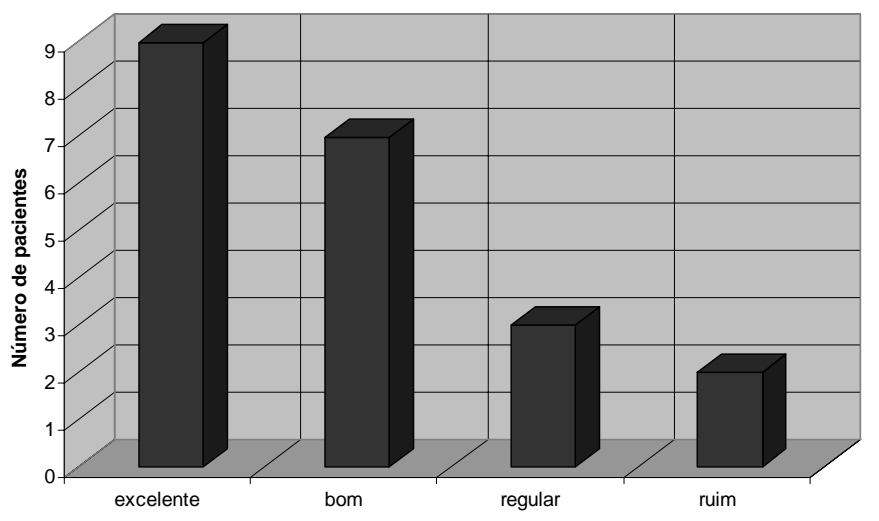

Gráfico 2 - Demonstração dos resultados segundo o sistema de pontuação de Bishop

Fonte: Universidade Federal de São Paulo - DOT - EPM 2008

\section{DISCUSSÃO}

A compressão do nervo ulnar no respectivo túnel é, em incidência, a segunda síndrome compressiva do membro superior. A primeira descrição de tratamento desta entidade data de 1816, quando se indicava a ressecção do segmento neural acometido ${ }^{(9-10)}$. Existem relatos de diversas possibilidades de tratamento, tanto cirúrgicas quanto conservadoras. O tratamento não cirúrgico consiste em fisioterapia, antiinflamatórios não hormonais, mudança das atividades e imobilização noturna com o cotovelo em flexão de $45^{\circ}(11)$. As opções de tratamento cirúrgico são diversas, iniciando pela neurólise do nervo ulnar ${ }^{(12)}$, transposição subcutânea ${ }^{(13-14)}$, transposição submuscular ${ }^{(15-16)}$, transposição intramuscular ${ }^{(8)}$ e a epicondilectomia parcial me$\operatorname{dial}^{(17-20)}$.

Uma das principais vantagens da neurólise do ulnar fundamenta-se na facilidade técnica do procedimento; todavia, é comum não obtermos sua plena liberação. Além do relaxamento neural, a transposição anterior (submuscular, subcutânea ou intermuscular) o posiciona em um local protegido de traumatismos e livre de aderências em todo arco de movimento. A desvantagem dessa abordagem encontra-se no risco de isquemia nervosa, secundária a manipulação excessiva, seguida de lesões intrínsecas ${ }^{(21)}$.

A compressão contra epicôndilo medial consiste em uma das causas mais relacionadas da síndrome do túnel cubital em virtude da fricção, do alongamento, da compressão e da tração do nervo ulnar por essa estrutura $^{(22)}$. A epicondilectomia medial parcial do cotovelo proporciona um leito adequado para a livre excursão do ulnar. Não obstante, esse procedimento é passível de diversas complicações, tais como: tendinopatias, luxação do nervo ulnar, perda da força de flexão e pronação, contratura em flexão e instabilidade em valgo. Em nosso estudo não obtivemos as complicações supracitadas.

Muermans et al(21) relataram que o paciente pode evoluir com dor residual no cotovelo devida a irritação no nervo ulnar por sua movimentação desprotegida. Encontramos essa dor residual em um paciente, porém de baixa intensidade, e não houve necessidade de procedimento adicional. A dor residual pode ser minimizada com a regularização das bordas do epicôndilo medial seguida da sutura minuciosa do periósteo. O único estudo prospectivo e randomizado, comparando a transposição anterior do nervo ulnar isolada com a transposição associada à epicondilectomia medial parcial, demonstrou superioridade desta ${ }^{(21)}$. 
No presente estudo, 17 pacientes $(80,9 \%)$ encontravam-se, segundo a classificação de McGowan, nos graus I e II. Os resultados cirúrgicos destes foram classificados como: nove excelentes $(42,8 \%)$, seis bons $(28,5 \%)$ e um regular $(4,7 \%)$, conforme a escala de Bishop $^{(8)}$. Apenas um paciente $(4,7 \%)$ apresentou resultado ruim no pós-operatório e este possuía, como antecedente, hanseníase.

Dos pacientes classificados no grau III de McGowan, um $(4,7 \%)$ apresentou resultado ruim, um $(4,7 \%)$, regular e outros dois $(9,4 \%)$, excelente. No subtipo III existe uma lesão intrínseca do nervo; assim, o resultado esperado está aquém do dos demais subgrupos.

\section{CONCLUSÃO}

Diante dos dados apresentados, concluímos que a neurólise do nervo ulnar associada à epicondilectomia parcial medial leva a aproximadamente $71 \%$ de bons e excelentes resultados, nos casos classificados como grau I e II de McGowan.

\section{REFERÊNCIAS}

1. Fernandes $\mathrm{CH}$, Matsumoto MH, Honmoto PK, Lima MF, Faloppa F, Albertoni WM, Carneiro R. Transposição anterior submuscular do nervo ulnar para o tratamento da síndrome do túnel cubital. Rev Bras Ortop. 1998;33(6):465-71.

2. Apfelberg DB, Larson SJ. Dynamic anatomy of the ulnar nerve at the elbow. Plast Reconstr Surg. 1973;51(1):79-81.

3. Osterman AL, Spiess AM. Medial epicondylectomy. Hand Clin. 2007;23(3):329-37.

4. Göbel F, Musgrave DS, Vardakas DG, Vogt MT, Sotereanos DG. Minimal medial epicondylectomy and decompression for cubital tunnel syndrome. Clin Orthop Relat Res. 2001;(393): 228-36.

5. Kaempffe FA, Farbach J. A modified surgical procedure for cubital tunnel syndrome: partial medial epicondylectomy. J Hand Surg [Am]. 1998;23(3):492-9.

6. King T. The treatment of traumatic ulnar neuritis; mobilization of the ulnar nerve at the elbow by removal of the medial epicondyle and adjacent bone. Aust N Z J Surg. 1950;20(1):3342.
7. McGowan AJ. The results of transposition of the ulnar nerve for traumatic ulnar neuritis. J Bone Joint Surg Br. 1950; 32-B(3):293-301.

8. Kleinman WB, Bishop AT. Anterior intramuscular transposition of the ulnar nerve. J Hand Surg [Am]. 1989; 14(6):972-9.

9. Adelaar RS, Foster WC, McDowell C. The treatment of the cubital tunnel syndrome. J Hand Surg [Am]. 1984;9A(1):90-5.

10. Macnicol MF. The results of operation for ulnar neuritis. J Bone Joint Surg Br. 1979;61-B(2):159-64.

11. Morrey B. The elbow and it's disorders. 3rd ed. Philadelphia: W.B. Saunders; 2000.

12. Sokolow C, Pariser P, Lemerle JP. [Ulnar nerve disorders at the elbow. Etiologic approach. Apropos of 40 cases] Ann Chir Main Memb Super. 1993;12(1):12-8.

13. Eaton RG, Crowe JF, Parkes JC 3rd. Anterior transposition of the ulnar nerve using a non-compressing fasciodermal sling. $\mathrm{J}$ Bone Joint Surg Am. 1980;62(5):820-5.

14. Foster RJ, Edshage S. Factors related to the outcome of surgically managed compressive ulnar neuropathy at the elbow level. J Hand Surg [Am]. 1981;6(2):181-92.

15. Leffert RD. Anterior submuscular transposition of the ulnar nerves by the Learmonth technique. J Hand Surg [Am]. 1982; 7(2):147-55.

16. Amadio PC, Beckenbaugh RD. Entrapment of the ulnar nerve by the deep flexor-pronator aponeurosis. J Hand Surg [Am]. 1986;11(1):83-7.

17. Goldberg BJ, Light TR, Blair SJ. Ulnar neuropathy at the elbow: results of medial epicondylectomy. J Hand Surg [Am]. 1989;14(2 Pt 1):182-8.

18. Heithoff SJ, Millender LH, Nalebuff EA, Petruska AJ Jr. Medial epicondylectomy for the treatment of ulnar nerve compression at the elbow. J Hand Surg [Am]. 1990;15(1):22-9.

19. Jones RE, Gauntt C. Medial epicondylectomy for ulnar nerve compression syndrome at the elbow. Clin Orthop Relat Res. 1979;(139):174-8.

20. Geutjens GG, Langstaff RJ, Smith NJ, Jefferson D, Howell CJ, Barton NJ. Medial epicondylectomy or ulnar-nerve transposition for ulnar neuropathy at the elbow? J Bone Joint Surg Br. 1996;78(5):777-9.

21. Muermans S, De Smet L. Partial medial epicondylectomy for cubital tunnel syndrome: Outcome and complications. J Shoulder Elbow Surg. 2002;11(3):248-52.

22. Gervasio O, Gambardella G, Zaccone C, Branca D. Simple decompression versus anterior submuscular transposition of the ulnar nerve in severe cubital tunnel syndrome: a prospective randomized study. Neurosurgery. 2005;56(1):108-17. 